\title{
Dommages des acides nucléiques induits après exposition aux rayonnements $X$ et UV
}

\author{
J. Cadet, M. Berger, T. Douki, D. Gasparutto, J.-P. Pouget, \\ J.-L. Ravanat et S. Sauvaigo
}

\author{
Laboratoire Lésions des Acides Nucléiques, SCIB, Département de Recherche Fondamentale \\ sur la Matière Condensée, CEA Grenoble, 38054 Grenoble, France
}

\begin{abstract}
Résumé : L'accent est mis dans ce court article de revue sur la description des principales réactions de modification photo- et radio-induite de la base guanine de l'ADN isolé et de composés modèles. Il est important de rappeler que la base guanine est le constituant le plus sensible aux réactions d'oxydation à un électron qui sont impliquées dans les processus de photosensibilisation de type I et l'effet direct du rayonnement ionisant. Ceci est complété par la présentation des principales réactions d'oxydation de la guanine par l'oxygène singulet (mécanisme de photosensibilisation de type II) et le radical hydroxyle (effet indirect du rayonnement ionisant). Enfin des exemples récents de mesure de dommages photo- et radio-induits de bases puriques et pyrimidiques dans l'ADN cellulaire sont présentés.
\end{abstract}

\section{INTRODUCTION}

Les effets biologiques des rayonnements ultraviolet et ionisant sur les organismes vivants s'expliquent principalement par une atteinte chimique des acides désoxyribonucléiques (ADN). La compréhension des mécanismes de dégradation photo- et radio-induite de l'ADN implique l'étude de systèmes modèles (ADN isolé, nucléosides, courts oligonucléotides). Ces travaux reposent sur l'attribution structurale des produits de modification [1-3] et les informations cinétiques et chimiques concernant les espèces radicalaires et excitées impliquées dans ces réactions [4,5]. L'action du rayonnement solaire sur l'ADN fait intervenir deux mécanismes principaux. L'interaction du rayonnement UVB (290 $\mathrm{nm}<\lambda<320 \mathrm{~nm}$ ) avec les bases de l'ADN se traduit essentiellement par la formation de photoproduits dimériques entre les bases pyrimidiques (cytosine et thymine). Ces derniers sont représentés par les cyclobutadipyrimidines de configuration cis-syn, les photoadduits pyrimidine (64) pyrimidone et leurs isomères de valence Dewar [2,3]. La structure de la plupart de ces photoproduits à l'exception de celle des photoadduits de cytosine est désormais bien établie. On peut aussi signaler, toujours par effet direct, la génération de photoproduits minotiraires qui implique les bases puriques (adénine et guanine). A titre d'exemples, on peut citer la formation d'un adduit entre l'adénine et la thymine ainsi que la production de 8-oxo-7,8-dihydroguanine (8-oxoGua) après ionisation de la base guanine [6]. Un deuxième mécanisme qui devient prédominant pour les régions UVA $(320 \mathrm{~nm}<\lambda<400 \mathrm{~nm})$ et visible $(\lambda>400 \mathrm{~nm})$ du spectre solaire est indirect. Il fait intervenir des agents de photosensibilisation endogènes (porphyrines, flavines) qui après excitation sont capables d'oxyder les bases de l'ADN et plus particulièrement la guanine par arrachement d'un électron ou d'un atome d'hydrogène selon un mécanisme de type I $[2,6]$. On peut aussi mentionner la possibilité de génération d'oxygène singulet par transfert d'énergie (mécanisme de type II). Ces diverses réactions de photooxydation sensibilisée de la base guanine qui ont fait l'objet de récents travaux seront décrites ultérieurement.

Les effets moléculaires du rayonnement ionisant sur l'ADN cellulaire s'expliquent par une double action $[1,4]$. Les processus directs font intervenir l'ionisation et l'excitation des bases ou du fragment osidique alors que les effets indirects sont associés à la radiolyse de l'eau avec production du radical hydroxyle et d'espèces réductrices (électron solvaté, atome d'hydrogène). Il en résulte différentes catégories de dommages dont les plus importantes sont les cassures simple et double brins d'ADN, les modifications de bases, les sites abasiques et les pontages ADN-protéines [7]. Il est important de préciser que l'action du rayonnement ionisant ne peut se résumer à la contribution d'événements radicalaires aléatoires donnant naissance à des modifications isolées. La notion d'événements multiples dont l'importance quantitative augmente avec le transfert d'énergie linéique (TEL) du rayonnement est à prendre en considération. La formation de cassures double-brin est 
l'illustration de la participation d'un processus dégradatif radio-induit de manière indépendante sur chacun des deux brins d'ADN à une distance inférieure à dix paires de bases. Pour cela, il a été proposé sur la base d'études théoriques que des réactions d'ionisation de l'ADN et des molécules d'eau environnantes sont susceptibles d'être générées le long de la trace d'un photon avec perte progressive de l'énergie de ce dernier [8]. Il est aussi possible d'envisager la formation d'autres types de lésions multiples associant deux ou éventuellement trois dommages (cassures, bases modifiées) sur le même brin ou sur les deux brins opposés [9]. Les principales réactions du radical hydroxyle avec les bases puriques et pyrimidiques de l'ADN isolé et de composés modèles sont désormais établies et les produits de modification qui en résultent ont été isolés et identifiés. Il en est de même pour les dommages qui résultent d'une oxydation à un électron. Les principaux mécanismes de dégradation de la guanine faisant intervenir d'un part les réactions d'addition du radical hydroxyle et d'autre part l'ionisation de cette base purique sont rapportés.

Cet article de revue est complété par la présentation de résultats récemment obtenus sur la mesure de dommages photo- et radio-induits dans de l'ADN cellulaire. Pour cela des méthodes de mesure sensibles et spécifiques ont été mises au point. Il faut rappeler que de manière générale la quantification de dommages individualisés de I'ADN et particulièrement celle des modifications de type oxydatif reste un défi analytique (vide infra).

\section{DOMMAGES PHOTO- ET RADIO-INDUITS DE LA GUANINE}

Comme cela a déjà été mentionné, l'essentiel des résultats d'études mécanistiques sur la formation de dommages photo- et radio-induits de l'ADN qui font l'objet de cet article concerne la base guanine (1).

\subsection{Réactions d'oxydation photosensibilisée}

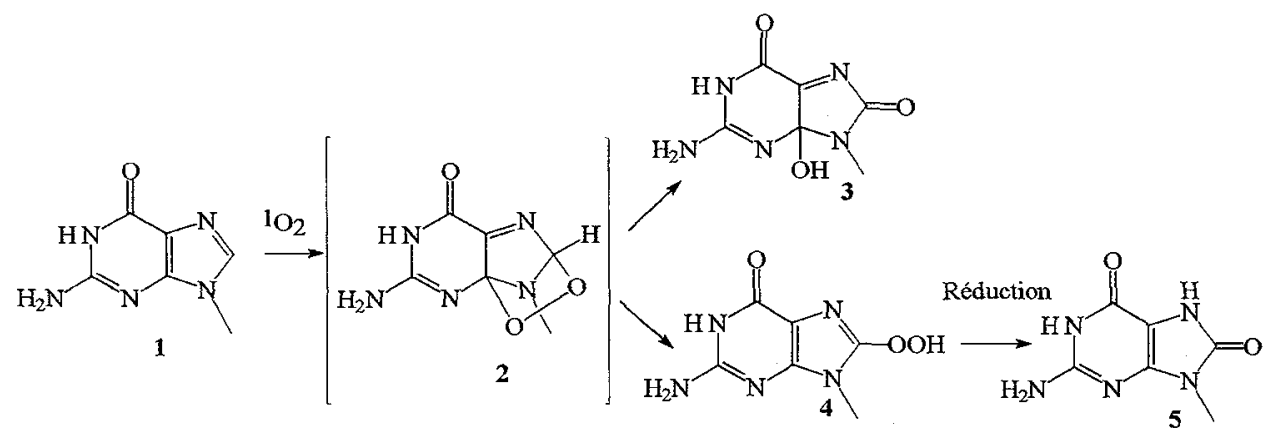

Figure 1 : Oxydation de la base guanine (1) par l'oxygène singulet.

Les réactions de photooxydation sensibilisée de la guanine (1) font intervenir un double mécanisme (type I et type II) dont l'importance relative dépend étroitement du photosensibilisateur [3]. Ainsi les phtalocyanines, le rose Bengale et le bleu de méthylène agissent de manière prépondérante avec 1 de manière indirecte par l'intermédiaire de l'oxygène singulet dans sa configuration $\Delta_{\mathrm{g} .}{ }^{1} \mathrm{O}_{2}\left(\Delta_{\mathrm{g}}\right)$ ainsi généré par transfert d'énergie est un puissant diénophile qui réagit avec les doubles liaisons riches en électrons. La principale réaction de ${ }^{1} \mathrm{O}_{2}\left(\Delta_{\mathrm{g}}\right)$ avec 1 est une cycloaddition [4+2] de type Diels-Alder impliquant les positions 4 et 8 du cycle purique (Fig. 1). La dégradation thermique des deux diastéréoisomères de l'endoperoxyde 2 instable qui en résulte pour la $2^{\prime}$-desoxyguanosine (dGuo) conduit aux formes $4 R$ et $4 S$ de la 8-oxo-4-hydroxy-4,8-dihydro-2'-désoxyguanosine (3) ou 8-oxo-4OHdGuo [10]. La 8-oxodGuo 5 qui résulte d'une transposition des endoperoxydes 2 en 8hydroperoxy-2'-désoxyguanosine (4) suivie d'une réduction de ce dernier intermédiaire est un produit minoritaire dans le cas du nucléoside et de courts fragments mono-brin de l'ADN $[6,10]$. Par contre, seule la 8-oxodGuo 5, à l'exclusion de 8-oxo-4-OHdGuo 3, est formée dans un ADN double-brin [11]. 


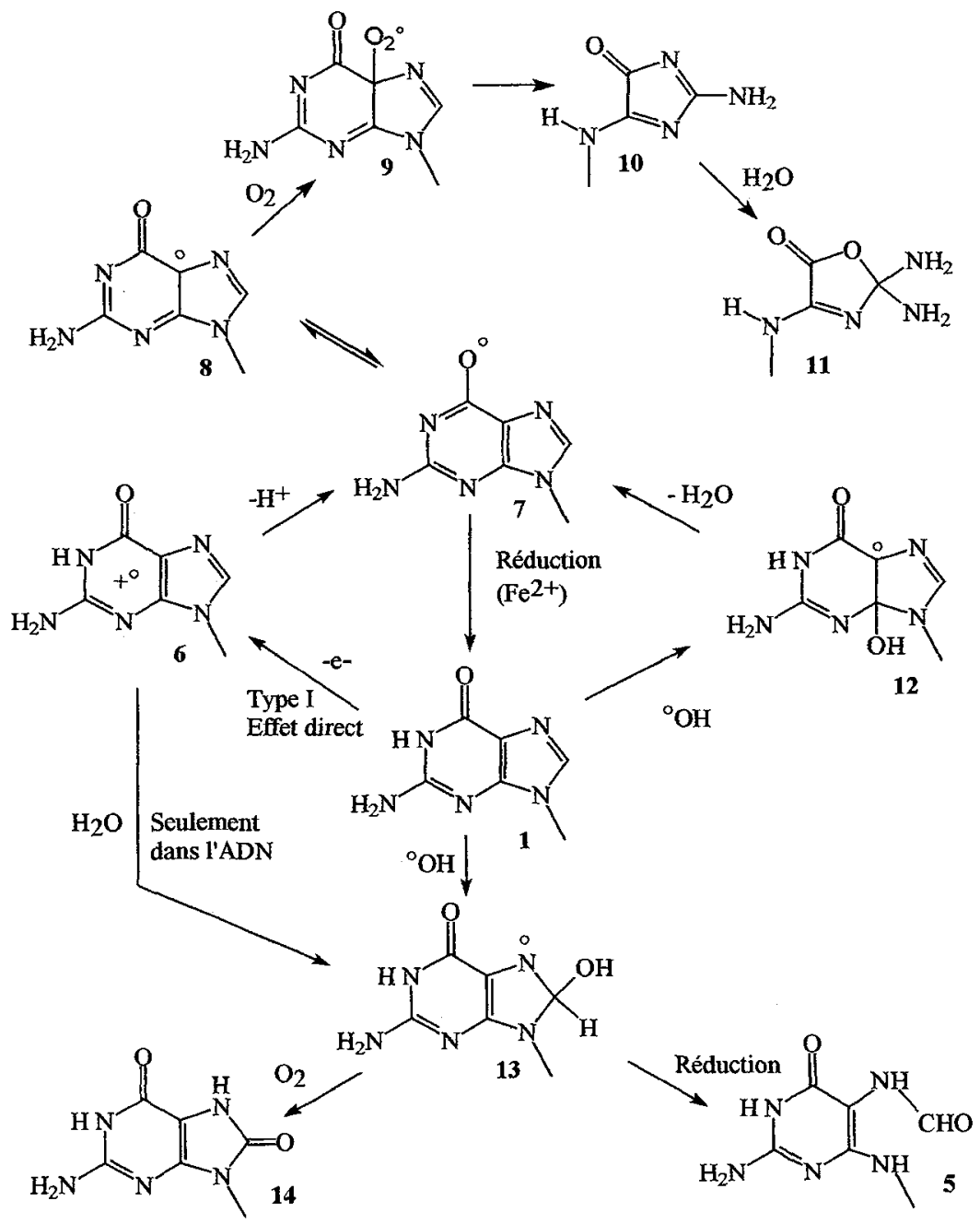

Figure 2 : Dégradation de la base guanine par oxydation à un électron et le radical ${ }^{\circ} \mathrm{OH}$.

La riboflavine et la benzophénone constituent deux exemples de photosensibilisateurs qui réagissent avec la base de dGuo 1 par arrachement d'un électron (type I). Le cation radical 6 (Fig. 2) qui en résulte se déprotone quantitativement pour générer le radical oxyle 7 fortement oxydant [5]. Ceci explique qu'un métal de transition sous forme réduite comme $\mathrm{Fe}^{2+}$ est capable de réduire 7 pour restaurer la molécule initiale 1 . La réaction de $\mathrm{O}_{2}$ avec 7 ou plutôt une de ses formes mésomères comme 8 qui présente un électron non apparié sur le carbone 5 conduit au radical peroxyle $9 . \mathrm{Ce}$ dernier après une série de transpositions donne lieu à la formation du dérivé de type imidazolone 10 [12-14] et à la libération de formylamine [15]. L'hydrolyse de $10\left(1 / 2\right.$ vie $=20 \mathrm{~h}$ à $20^{\circ} \mathrm{C}$ et à $\left.\mathrm{pH} \mathrm{7}\right)$ engendre l'oxazolone 11 qui est la lésion sensible au traitement à chaud par la pipéridine alors que les oligonucléotides contenant un résidu 8-oxodGuo 5 sont stables dans ces mêmes conditions [16-18].

L'hydratation du cation radical de la guanine 6 qui n'est pas détectable dans un nucléoside ou un court oligonucléotide devient une réaction d'importance dans l'ADN double-brin $[19,20]$. Il a été ainsi montré que l'hydratation de 6 dans de l'ADN de thymus de veau conduit à la formation de 8oxodGuo 5 via le radical 8-hydroxy-7,8-dihydropuryle 13. On note aussi la formation du dérivé 2,6diamino-4-hydroxy-5-formamidopyrimidine (14) ou FapyGua par réduction du radical 13 suivie de l'ouverture du cycle imidazole [21] (Fig. 2). 


\subsection{Effets directs et indirects des rayonnements ionisants}

L'ionisation (effet direct) de la molécule de guanine (1) conduit à la formation du cation radical purique 6. La chimie de transformation de cet intermédiaire très réactif est identique à celle décrite pour la décomposition de 1 par réaction photosensibilisée de type I [7] (Fig. 2).

L'effet indirect du rayonnement ionisant sur 1 en solution aqueuse aérée se résume principalement à l'action du radical $\mathrm{OH}$ formé par radiolyse de l'eau. On peut signaler que les espèces réductrices (électron solvaté $H$ ) n'ont pas d'effets endommageants puisqu'elles sont captées directement ou indirectement par $\mathrm{O}_{2}$. Deux sites principaux d'addition du radical $\mathrm{OH}$ ont été proposés [4,5]. L'addition de $\mathrm{OH}$ sur le carbone $\mathrm{C} 5$ de 1 conduit à la formation de l'oxazolone 11 via son précurseur imidazolone 10 et le radical oxyle $7[7,22]$. Ce dernier est généré par déshydratation du radical 12. Le carbone 8 du cycle imidazole constitue un deuxième site d'addition du radical $\mathrm{OH}$ (Fig. 2). Comme cela a été décrit précédemment, la transformation en solution aqueuse aérée du radical 8-hydroxy-7,8-dihydroguanyle 13 conduit dans l'ADN double-brin à la production prépondérante de 8-oxoGua 5 avec une faible quantité de dérivé FapyGua 14 [22,23].

\section{MESURE DE DOMMAGES PHOTO- ET RADIO-INDUITS DES BASES PURIQUES ET PYRIMIDIQUES DANS L'ADN CELLULAIRE}

La recherche de modifications photo- et radio-induites des bases de l'ADN cellulaire reste un défi analytique qui s'est heurté au cours de la dernière décennie à divers problèmes méthodologiques [2426]. Il faut rappeler que le niveau de sensibilité qui doit être atteint est d'environ 1 modification de type oxydatif pour $10^{6}$ bases normales dans un échantillon d'ADN de l'ordre de 20 à $30 \mu \mathrm{g}$. L'application de la méthode de chromatographie en phase gazeuse avec une détection par spectrométrie de masse (CG-SM) [27-29] a conduit à l'obtention de résultats erronés avec une surestimation des résultats d'au moins un facteur 20 concernant la mesure des bases oxydées [30-33]. Ceci est dû à une oxydation artéfactuelle des bases normales au cours de la réaction de dérivation (sylilation) [34-36] qui est nécessaire pour rendre les molécules volatiles avant l'analyse CG. Il faut ajouter la possibilité d'oxydation des bases normales majoritaires au cours de l'extraction de l'ADN de la cellule et des étapes suivantes de purification et de digestion [25,26]. Ces réactions d'autooxidation qui sont désormais en grande partie prévenues par en particulier l'utilisation d'une méthode d'extraction chaotropique [37] donnent lieu à une augmentation d'un facteur 5 à 10 du taux basal de 8-oxodGuo 5. En fait, seul ce dernier nucléoside oxydé a été mesuré dans l'ADN cellulaire après exposition au rayonnement ionisant en utilisant la chromatographie liquide associée à une détection électrochimique [38]. Le taux de formation de 5 n'a pu être déterminé qu'à des doses élevées (> $100 \mathrm{~Gy}$ ) en raison de l'existence d'un bruit de fond artéfactuel. Toutefois l'utilisation conjointe de la méthode des comètes et d'enzymes de réparation (endonucléase III et protéine Fpg) [39] qui convertissent en coupures de brins additionnelles des bases modifiées pyrimidiques et puriques respectivement a permis de surmonter ces difficultés. Ainsi des dommages radio-induits ont été mesurés dans un domaine de doses compris entre 2 et 10 Gy ( 738 coupures et sites alcali-labiles, 294 bases reconnues par l'endonucléase III et 264 sites sensibles à l'enzyme Fpg par cellule et par Gy) [38]. Il faut aussi signaler l'émergence de deux nouvelles méthodes sensibles et spécifiques qui devraient recevoir de nombreuses applications dans un proche avenir. L'association de l'électrophorèse capillaire à une détection laser par immunofluorescence a permis de mesurer la formation de 0,9 résidu de 5,6-dihydroxy-5,6-dihydrothymine pour $10^{7}$ bases dans 1'ADN cellules humaines cancéreuses de poumon après exposition à 1 Gy [40]. Une autre approche consiste à associer la chromatographie liquide haute performance avec une détection par spectrométrie de masse (ionisation par "electrospray") en mode tandem [41]. Le niveau de détection de 8-oxodGuo 5 est dans ces conditions voisin de la femtomole. On peut ajouter que l'utilisation de la méthode des comètes avec une détection par immunofluorescence présente de grandes potentialités pour la mesure de lésions oxydatives de l'ADN dès lors que l'on possède des anticorps hautement spécifiques. Ceci n'est pas encore la cas pour les bases oxydées de l'ADN. Par contre la mesure du 
dimère cis-syn de cyclobutadithymine a été effectuée dans de l'ADN de cellules fibroblastiques humaines après exposition à $0,5 \mathrm{~kJ} / \mathrm{m}^{2}$ de rayonnement $\mathrm{UVB}$ en utilisant une approche similaire [42]

\section{Références}

1. J. Cadet, M. Berger, Int. J. Radiat. Biol. 74, 127 (1985).

2. J. Cadet, P. Vigny, in Bioorganic Photochemistry: Photochemistry and the Nucleic Acids, edited by H. Morrison (Wiley \& Sons, New York, 1990) Vol. 1, p.1.

3. J. Cadet, in DNA adducts: Identification and biological significance, edited by K. Hemminki et al. (IARC Scientific Publications, Lyon, 1994) No. 125, p. 245

4. C. von Sonntag, in The Chemical Basis of Radiation Biology (Taylor \& Francis, London, (1987)

5. S. Steenken, Chem. Rev. 89, 503 (1989).

6. J. Cadet, M. Berger, T. Douki, B. Morin, S. Raoul, J.-L. Ravanat, S. Spinelli, Biol. Chem. 378, 1275 (1997).

7. J. Cadet, M. Berger, T. Douki, J.-L. Ravanat, Rev. Physiol. Biochem. Pharmacol. 131, 1 (1997)

8. H. Nikjoo, P. O'Neill, M. Terrissol, D. T. Goodhead, Int. J. Radiat. Biol. 66, 453 (1994).

9. J.-F. Ward, Int. J. Radiat. Biol. 66, 427 (1994),

10. J.-L. Ravanat, J. Cadet, Chem. Res. Toxicol. 8, 379 (1995).

11. J. Cadet, J,-L. Ravanat, G.W. Buchko, H.C. Yeo, B.N. Ames, Methods Enzymol. 234, 79 (1994)

12. J. Cadet, M. Berger, G.W. Buchko, P.C. Joshi, S. Raoul, J.-L. Ravanat, J. Am. Chem Soc. 116, 7403 (1994)

13. G.W. Buchko, J. Cadet, B. Morin, M. Weinfeld, Nucleic Acids Res. 19, 3954 (1995).

14. S. Raoul, M. Berger, G.W. Buchko, P.C. Joshi, B. Morin, M. Weinfeld, J. Cadet, J. Chem. Soc. Perkin Trans. 2, 371 (1996).

15. C. Vialas, G. Pratviel, C. Claparols, B. Meunier, J. Am. Chem. Soc. 120, 11548 (1998).

16. A. Spassky, D. Angelov, Biochemistry 36, 6571 (1997).

17. K. Kino, I. Saito, H. Sugiyama, J. Am. Chem. Soc. 120, 7373 (1998).

18. D. Gasparutto, J.-L; Ravanat, O. Gerot, J. Cadet, J. Am. Chem. Soc. 120, 10283 (1998).

19. H. Kasai, Z. Yamaizumi, M. Berger, J. Cadet, J. Am. Chem. Soc. 114, 9692 (1992).

20. D. Angelov, A. Spassky, M. Berger, J. Cadet, J. Am. Chem. Soc. 119, 11373 (1997).

21. T. Douki, J. Cadet, Int. J. Radiat. Biol. (in press).

22. T. Douki, J. Cadet, Free Rad. Res. 24, 369 (1996)

23. T. Douki, R. Martini, J.-L. Ravanat, R.J. Turesky, J. Cadet, Carcinogenesis 18, 2385 (1997).

24. J. Cadet, M. Weinfeld, Anal. Chem. 65, 675A (1993).

25. J. Cadet, T. Douki, J.-L. Ravanat, Environ. Health Perspect. 105, 1034 (1997).

26. A. Collins, J. Cadet, B. Epe, C. Gedik, Carcinogenesis 18, 1833 (1997).

27. M. Dizdaroglu, Free Radical Biol. Med. 10, 225 (1991).

28. B. Halliwell, M. Dizdaroglu, Free Rad. Res. Commun. 16, 15 (1992).

29. M. Dizdaroglu, FEBS Lett. 315, 1 (1993).

30. I.D. Podmore, H.R. Griffiths, K.E. Herbert, N. Mistry, P. Mistry, J. Lunec, Nature, 392, 559 (1998)

31. M.-L. Kuo, S.-H. Jee, M.-H. Chou, T.-H. Ueng, Mutat. Res. 413, 143 (1998).

32. T.H. Zastawny, M. Kruszewski, R. Olinski, Free Radical Biol. Med. 24, 1250 (1998).

33. V. Abalea, J. Cillard, M.-P. Dubos, J.-P. Anger, P. Cillard, I. Morel, Carcinogenesis 19, 1053 (1998)

34. J-L. Ravanat, R.J. Turesky, E. Gremaud, L.J. Trudel, R.H. Stadler, Chem. Res. Toxicol. 8, 1039 (1995)

35. T. Douki, T. Delatour, F. Bianchini, J. Cadet, Carcinogenesis 17, 347 (1996).

36. H.E. Poulsen, A. Weimann, J.T. Salonen, K. Nyyssönen, S. Loft, J. Cadet, T. Douki, J.-L. Ravanat, Nature 395, 231 (1998).

37. H.J. Helbock, K.B. Bechman, M.K. Shigenaga, P.B. Walker, A.A. Woodhall, H.C. Yeo B.N. Ames, Proc. Natl. Acad. Sci. USA 95, 283 (1998).

38. J.-P. Pouget, J.-L. Ravanat, T. Douki, M.-J. Richard, J. Cadet, Int. J. Radiat. Biol (in press).

39. A.R. Collins, M. Dusinska C.M. Gedik, R. Stetina, Environ. Health Perspect.104, 465 (1996).

40. X.C. Le, J.Z. Xing, J. Lee, S.A. Leadon, M. Weinfeld, Science 280, 1067 (1998).

41. J.-L. Ravanat, B. Duretz, A. Guiller, T. Douki, J. Cadet, J. Chromatogr. B 715, 349 (1998).

42. S. Sauvaigo, C. Serres, N. Signorini, N. Emonet, M-J. Richard, J. Cadet, Anal. Chem. 259, 1 (1998). 